\title{
Nanonstructural Analysis of Hydroxyapatite Thin Films using HRTEM/FIB Techniques
}

\author{
E. L. Solla*, B. Rodríguez-González*, I. Pereiro**, C. Rodríguez-Valencia**, B. C. Cores**, J.
}

Serra** and P. González**

* Servicio de Microscopía Electrónica, CACTI, Universidade de Vigo, As Lagoas-Marcosende, 36310

Vigo, Spain

** Departamento de Física Aplicada, Universidade de Vigo, As Lagoas-Marcosende, 36310 Vigo, Spain

Hydroxyapatite, with a composition that resembles the mineral phase of bone, is often used as a coating of metallic prostheses to improve the adhesion and osseointegration of implants in different parts of the skeleton [1]. Among the available techniques that allow the deposition of this material on metallic substrates, Pulsed Laser Deposition (PLD) is capable of producing high quality and highly biocompatible coatings with excellent final attachment [2,3].

In the present work, the hydroxyapatite coatings were deposited on titanium substrates by PLD, keeping the substrate temperature at $460{ }^{\circ} \mathrm{C}$ and carrying the process under a low-pressure water vapour atmosphere. In order to study the nanostructure of the coatings by HRTEM, a cross section thin specimen was prepared by FIB.

The FIB lift out site and its resulting thin lamella are depicted on Figure 1. The SAED pattern presented on Figure 2 is consistent with a nanocrystalline hydroxyapatite structure, where many reflections from lattice planes such as (002), (121) or (120) were recorded. The dark field images were obtained selecting the diffracted beams of the (002) reflection, clearly showing the columnar arrangement of the 002 oriented nanocrystals from the substrate to the coating surface. Finally, the HRTEM analysis (Figure 3) indicated an abundance of nanocrystals with an interplanar distance of $3.44 \AA$, corresponding to the distance between 002 planes present in the hydroxyapatite crystal structure.

\section{References}

1. Suchanek et al., Journal of Materials Research 13: 94-117, 1998

2. Torrisi et al., Thin Solid Films 227: 32-36, 1993

3. Peraire et al., Journal of Biomedical Materials Research 77ª: 370 -379, 2006 

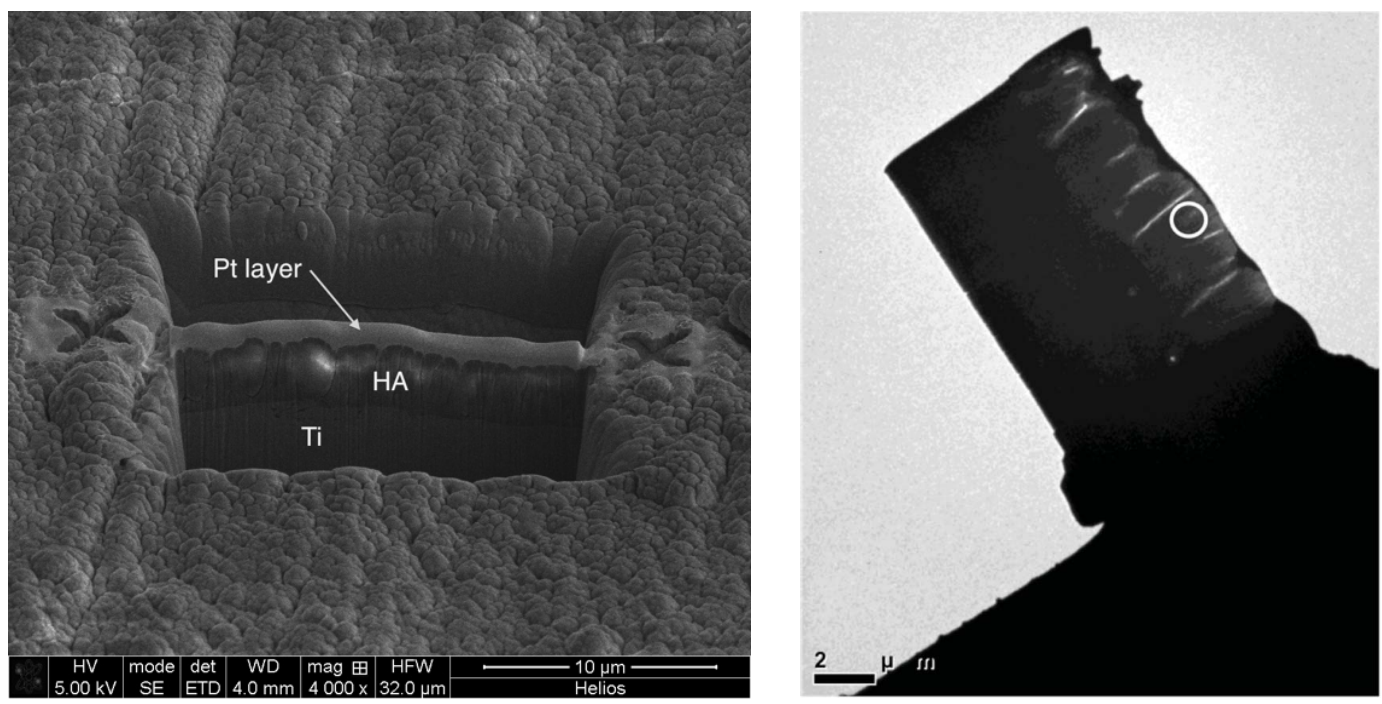

Figure 1. Thin lamella preparation using the in situ lift out technique. The HA coating, Ti substrate and Pt protective layer are clearly visible. In the low magnification TEM micrograph of the thin lamella, the circled area is where the SAED pattern presented on Fig. 2 was obtained.
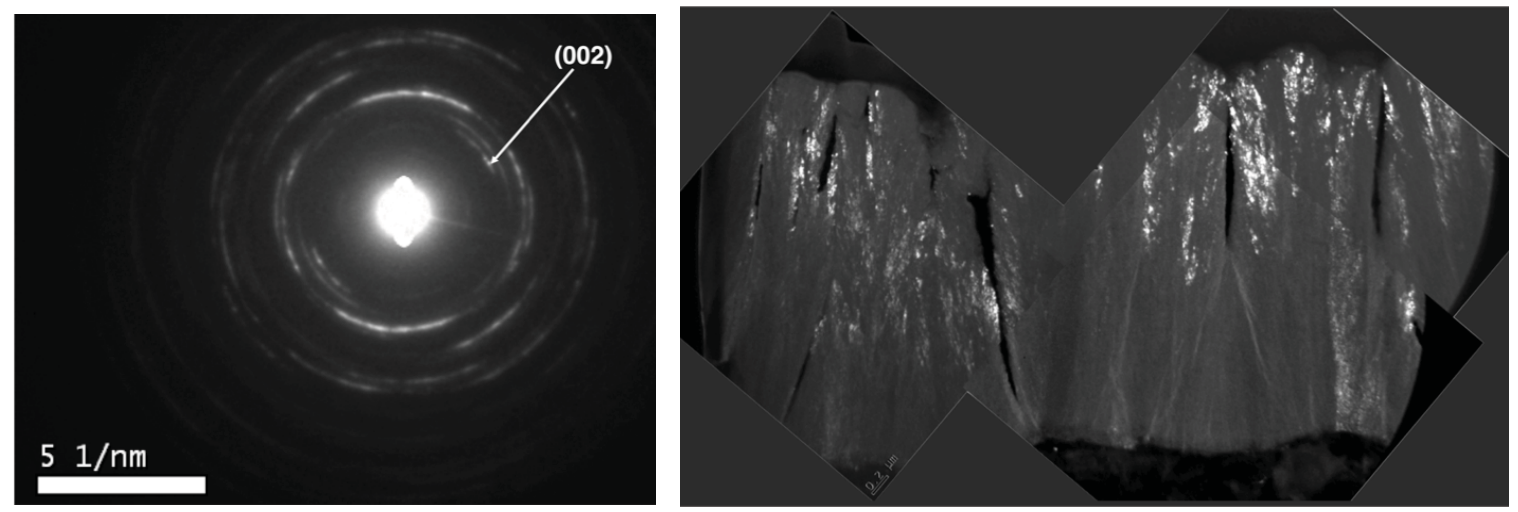

Figure 2. SAED pattern of the nanocrystalline HA present in the coating, showing the (002) reflection selected to form the dark field images. The composition of three dark field images comprises the whole thickness of the coating.

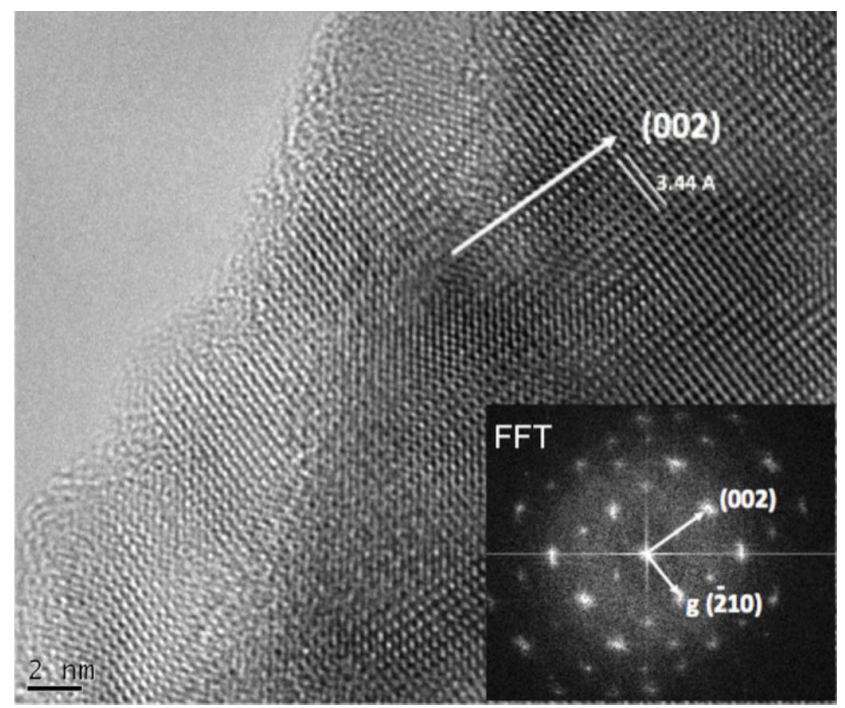

Figure 3. HRTEM micrograph of a hydroxyapatite nanocrystal viewed from the $<120>$ zone axis. The interplanar distance distance corresponds to the 002 plane of hydroxyapatite. 